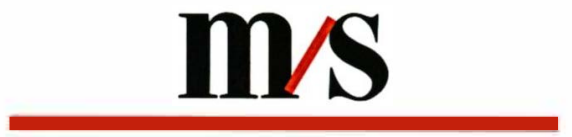

médecine/sciences $1995 ; 11: 1067-9$

\title{
L'AVENIR THÉRAPEUTIQUE DES FACTEURS NEUROTROPHIQUES DANS LES MALADIES NEURO-DÉGÉNÉRATIVES
}

es maladies neuro-dégénératives représentent un problème majeur de santé publique. Des maladies génétiques de l'enfant - comme les amyotrophies spinales infantiles - à celles souvent sporadiques de l'adulte, telle la maladie d'Alzheimer, la liste est longue d'affections dont l'étiopathogénie reste dans la plupart des cas à déterminer, et pour lesquelles les traitements disponibles sont peu efficaces face à l'évolution souvent rapide et foudroyante de la maladie.

Les facteurs neurotrophiques constituent un groupe désormais assez disparate de facteurs protéiques qui ont en commun une capacité de favoriser la survie neuronale dans différents modèles expérimentaux menant à la mort d'une classe donnée de neurones. Leur découverte a rapidement fait émerger l'idée de leur utilisation pour le traitement de maladies neuro-dégénératives. On a ainsi défini une "stratégie neurotrophique": l'emploi de ces facteurs naturels et pléiotropes pour aider les neurones à résister au processus pathologique, même lorsque celui-ci n'implique aucune altération du fonctionnement normal des facteurs euxmêmes [1].

La maladie sur laquelle est concentrée une grande partie des recherches cliniques dans ce domaine est la sclérose latérale amyotrophique
(SLA). La SLA, qui commence généralement vers l'âge de 50 ans, est caractérisée par une perte importante et progressive de motoneurones dans la moelle épinière et dans le cortex moteur. Plusieurs explications de la grande concentration de moyens sur cette affection peuvent être données. Premièrement, l'évolution rapide et inéluctable de la SLA, conjuguée à l'absence totale de traitement, crée une attente de la part des malades et des médecins qui facilite le démarrage d'essais rapides et novateurs. Deuxièmement, la liste de facteurs neurotrophiques capables de protéger le motoneurone spinal in vivo et in vitro ne cesse de s'allonger. Enfin, le motoneurone spinal, seul parmi les grandes classes de neurones centraux, se projette à la périphérie et est capable d'y capter et de transporter de façon rétrograde les facteurs neurotrophiques. Cette dernière considération a été déterminante lorsqu'il s'agissait de procéder à l'essai de facteurs protéiques ne traversant pas la barrière hémato-encéphalique.

Parmi les facteurs capables de favoriser la survie du motoneurone, on retrouve des membres de plusieurs grandes familles : les neurotrophines BDNF, NT-3 et NT-4/5, les cytokines CNTF et LIF, ainsi que des facteurs de croissance tels le FGF-2, le FGF-5, l'IGF-1, le GDNF, ou le TGFB3 (pour revue ainsi que pour la traduction de
C. Henderson : directeur de recherche au Cnrs, directeur de l'U.382 de l'Inserm. Inserm U.382 IBDM, Campus de Luminy, Case 907, 13288 Marseille Cedex 09, France. $m / s n^{\circ} 8$, vol. 11 , août 95 
sigles souvent caducs, voir [2-5]). Chacun devient de ce fait candidat potentiel pour le traitement de la SLA. Si le CNTF (ciliary neurotrophic factor) a été le premier à être testé, c'est en raison de considérations historiques et de propriété industrielle, mais également de l'existence dans la littérature de résultats précliniques à première vue impressionnants.

Ainsi, le CNTF recombinant appliqué à l'embryon de poulet pendant la période de mort cellulaire programmée permet de sauver environ un tiers des motoneurones qui sont perdus habituellement. Par ailleurs, le CNTF protège l'ensemble des motoneurones du noyau facial de la mort induite par axotomie chez le raton nouveau-né. De manière plus frappante encore, la durée de vie de la souris mutante pmn (progressive motor neuronopathy) est significativement prolongée lorsqu'elle reçoit des greffes de lignées cellulaires sécrétant des quantités importantes de CNTF [6]. Il est intéressant de souligner que, chez ce modèle murin, aucune altération du système CNTF/CNTF-récepteur n'a été décelée.

Sur la base de ces données, plusieurs essais cliniques du CNTF dans la SLA ont été entrepris, utilisant une in jection sous-cutanée quotidienne à différentes doses. Ces essais ont mis en évidence des effets secondaires importants aux plus fortes doses, tandis qu'à des doses plus faibles, mieux tolérées, aucun effet bénéfique n'a encore été rapporté. Les vrais problèmes auxquels se confrontaient ces essais commencent désormais à être ouvertement discutés. Certains de ceux-ci sont généralisables à l'ensemble des facteurs neurotrophiques, et seront évoqués plus loin, mais d'autres sont peut-être particuliers au CNTF. Par exemple, le CNTF peut se lier à un récepteur soluble qui est abondant dans le sérum humain. Sous cette forme, il peut agir au niveau des récepteurs d'une autre cytokine, le LIF (leukemia inhibitory factor), et induire des réactions inflammatoires indésirables [7]. Par ailleurs, la durée de vie du CNTF dans la circulation est brève, nécessitant l'utilisation chez les souris pmn n'avait pas été reproduit chez l'homme. Enfin, le CNTF à doses plus fortes entraîne chez l'animal une cachexie, voire la mort. Il s'agit donc de problèmes réels qui appellent des solutions avant tout techniques.

Un autre facteur dont l'essai clinique s'est déroulé en partie en parallèle est l'IGF-1 (insulin-like growth factor-1). Cette molécule a des effets trophiques au niveau du muscle et du motoneurone qui sont réels mais plus discrets que ceux d'autres facteurs [9].

Cependant, son absence de toxicité chez l'homme, ainsi que son effet simultané sur le neurone et le muscle ont encouragé un essai dans la SLA. La première analyse des résultats de l'essai américain n'est pas encore publiée, mais un communiqué de presse du 10 juin 1995 fait état d'observations prometteuses, notamment d'un ralentissement de la vitesse d'évolution de la maladie chez les patients traités, mais il faudra attendre la publication de ces résultats après expertise pour les juger.

Plus récemment encore (26 juin 1995), les résultats préliminaires d'un essai Phase I/II de la neurotrophine BDNF étaient rapportés dans la presse. Le BDNF augmenterait la capacité respiratoire mais, sur 283 patients, n'aurait pas d'effet significatif sur la durée de vie. Les effets secondaires étant mineurs, la Phase III doit débuter en 1995.

Quel est donc l'avenir de la stratégie neurotrophique dans les maladies neuro-dégénératives en général, et dans la SLA en particulier? Pour l'évaluer, il est nécessaire de mesurer les obstacles qui restent encore à franchir, et de tenir compte de l'avancée récente d'autres stratégies thérapeutiques (pour une revue plus détaillée, voir [10]). Vraisemblablement, le plus grand problème sera l'incapacité des facteurs neurotrophiques à franchir la barrière hémato-encéphalique, et la faible diffusion de certains d'entre eux dans le tissu nerveux. Cela est évident pour la maladie d'Alzheimer, par exemple, mais même dans le cas de la SLA, rien ne permet d'affirmer qu'une thérapeutique efficace des motoneurones spinaux corrigera l'atteinte des motoneurones corticaux, et donc une partie importante des symptômes. Plusieurs tentatives sont actuellement en cours pour contourner ce problème: implantation de cellules encapsulées dans le système nerveux central, installation de micro-pompes, injection dirigée d'adénovirus recombinants... De leurs résultats dépendra en grande partie la seconde génération d'essais chez l'homme.

Une autre considération importante est la durée nécessairement longue de tout traitement réussi. Cela s'accompagnera presque inévitablement d'une réponse immunitaire, mais il se peut qu'une administration intrathécale et lente minimise ce problème. Par ailleurs, il faudra s'assurer que l'effet biologique du facteur neurotrophique est maintenu. Or, après axotomie chez le raton nouveau-né, plusieurs facteurs administrés en bolus maintiennent les motoneurones pendant une semaine, mais aucun n'est efficace à deux semaines [11]. Il est à espérer que les techniques sus-citées, permettant la libération d'un taux faible mais constant de facteur neurotrophique, apporteront une solution partielle à ce problème en réduisant les problèmes de désensibilisation.

Il est évident qu'une petite molécule non protéique ayant la puissance, l'efficacité et la spécificité de certains facteurs représenterait la solution idéale pour contourner la majeure partie de ces obstacles. Une telle molécule pourrait être un agoniste d'un récepteur de facteur neurotrophique ou, au contraire, agir plus directement pour bloquer le processus pathologique, par exemple en protégeant contre des mécanismes excitotoxiques. Un exemple des premiers est le CEP 1347 développé par Cephalon, analogue de K252a, pour lequel une bonne activité protectrice des motoneurones est signalée. Parmi les seconds, il faut vraisemblablement compter le riluzole, développé par Rhône-Poulenc Rorer [12], dont l'essai à grande échelle dans la SLA vient d'être terminé, et qui semble, d'après les informations non expertisées dont nous disposons, permettre une prolongation de la vie des malades. 
Il est trop tôt pour savoir si une petite molécule de ce type pourra un jour retarder de façon complète l'évolution d'une maladie neurodégénérative. Il semble plus raisonnable de supposer qu'à moyen terme il faudra combiner un certain nombre de traitements dont chacun est partiellement efficace. La prise de participation dans les sociétés de biotechnologie par les grands de l'industrie pharmaceutique risque de ce point de vue d'avoir des eff ets positifs, tout au moins à moyen terme. D'une part, cela donnera une stabilité financière suffisante aux chercheurs pour que le besoin de trouver des investisseurs nouveaux ne soit pas à lui seul une motivation pour commencer un essai clinique. D'autre part, cela devrait favoriser les essais conjoints de petites molécules et de facteurs protéiques, ce qui semble être la voie la plus prometteuse à l'heure actuelle.

\section{RÉFÉRENCES}

1. Henderson CE. Applications potentielle des neurotrophines dans les affections dégénératives du motoneurone. In : Payen G, ed Stratégie des thérapeutiques innovantes. Paris : Frison-Roche, 1993 : 229-41.

2. Johnson J, Oppenheim RW. Keeping track of changing neurotrophic theory. Curr Biol $1994 ; 4: 662-5$.

3. Snider WD. Functions of the neurotrophins during nervous system development what the knockouts are teaching us. Cell $1994 ; 77: 627-38$

4. Henderson CE, Bloch-Gallego E, Camu W, et al. Neurotrophic factors in developmen and plasticity of spinal neurons. Restor Neurol Neurosa $1993 ; 5: 15-28$

5. Henderson CE, Phillips HS, Pollock RA, $e$ al. GDNF : a potent survival factor for motoneurons present in peripheral nerve and muscle. Science $1994 ; 266: 1062-4$.

6. Sagot Y, Tan SA, Baetge E, et al. Polymerencapsulated cell lines genetically engineered to release ciliary neurotrophic factor can slow down progressive motor neuronopathy in the mouse. Eur J Neurosci $1995 ; 7: 1313-22$.
7. Davis S, Aldrich TH, Ip NY, et al. Released form of CNTF receptor-alpha component as a soluble mediator of CNTF responses. Science 1993 ; 259 : 1736-9.

8. Sendtner M, Schmalbruch H, Stöckli KA, et al. Ciliary neurotrophic factor prevents degeneration of motor neurons in mouse mutant progressive motor neuronopathy. Nature $1992 ; 358$ : 502-4.

9. Lewis ME, Neff NT, Contreras PC, et al. Insulin-like growth factor-1 : potential for treatment of motor neuronal disorders. Exp Neurol $1993 ; 124: 73-88$.

10. Henderson CE. Neurotrophic factors as therapeutic agents in ALS. Adv Neurol 1995 ; $68: 235-40$

11. Vejsada R, Sagot Y, Kato AC. Quantitative comparison of the transient rescue effects of neurotrophic factors on axotomised motoneurons in vivo. EurJ Neurosci 1995 ; 7 : 108-15.

12. Bensimon G, Lacomblez L, Meininger V, et al. A controlled trial of riluzole in amyotrophic lateral sclerosis. $N$ Engl J Med 1994 ; 330 : 585-91

\section{TIRÉS À PART}

C. Henderson. 